\title{
CONTROL DE MALEZAS EN CAÑA DE AZÚCAR CON CLOMAZONE Y AMETRINA 1
}

\author{
Valentín A. Esqueda 2
}

\section{RESUMEN}

Control de malezas en caña de azúcar con clomazone y ametrina. Se establecieron dos experimentos para evaluar el efecto del herbicida clomazone aplicado en preemergencia y de la mezcla de clomazone + ametrina en postemergencia en el control de malezas y la toxicidad a la caña de azúcar. El clomazone tuvo un control de Echinochloa colona, Panicum fasciculatum y Lagascea mollis superior al $90 \%$ en dosis de 0,96 a $1,2 \mathrm{~kg} / \mathrm{ha}$, pero fueron necesarios $1,44 \mathrm{~kg} / \mathrm{ha}$ para obtener controles de $90 \%$ de Rottboellia cochinchinensis y entre 80 y $90 \%$ de Phyllanthus niruri y no tuvo efecto sobre Cyperus rotundus. La aplicación postemergente de clomazone+ametrina desde $800+1200 \mathrm{~g} / \mathrm{ha}$, tuvo controles de malezas semejantes o superiores a los de las mezclas de 2,4-D+Ametrina y 2,4-D+diurón, utilizados comúnmente en la región.El clomazone aplicado en postemergencia, ocasionó un blanqueamiento temporal del follaje de la caña de azúcar, el cual desapareció entre los 30 y 60 días después de la aplicación.

\section{ABSTRACT}

Weed control in sugarcane with clomazone and ametryn. Two experiments were carried out in order to evaluate the effect of the herbicide clomazone applied in preemergence and the mixture clomazone+ametryn in postemergence on weed control and toxicity to sugarcane. Clomazone from 0.96 to $1.2 \mathrm{~kg} / \mathrm{ha}$ controlled more than $90 \%$ of Echinochloa colona, Panicum fasciculatum and Lagascea mollis, but it was necessary to increase the dose to $1.44 \mathrm{~kg} / \mathrm{ha}$ to obtain a $90 \%$ control of Rottboellia cochinchinensis and between 80 and $90 \%$ of Phyllanthus niruri and it had no effect on Cyperus rotundus. The postemergent application of clomazone + ametryn from $800+1200 \mathrm{~g} / \mathrm{ha}$, showed weed control similar or superior to those of the mixtures of 2,4-D+ ametryn and diuron+2,4-D, which are commonly utilized in the region. A temporal whitening of the sugarcane foliage occurred when clomazone was applied in postemergence, but this symptom disappeared 30 and 60 days after the application.

\section{INTRODUCCIÓN}

En el estado de Veracruz, anualmente se dedican alrededor de 220,000 ha al cultivo de la caña de azúcar, siendo uno de los principales cultivos, tanto agronómica como socialmente, ya que desde el establecimiento hasta la cosecha, requiere una gran cantidad de mano de obra, por lo que genera un aporte económico importante no sólo entre los habitantes de las zonas cañeras, sino en otras localidades dentro y fuera del estado.

Una de las prácticas que más influyen para la obtención de una buena producción en caña de azúcar, es el control de las malezas, ya que éstas se presentan en grandes poblaciones sobre todo en las etapas iniciales de desarrollo de este cultivo y si no son controladas oportuna y eficientemente, pueden causar reducciones en la producción de hasta un 60\% (Díaz Calderón y Masson Colar, 1996).

Los herbicidas más utilizados en caña de azúcar, son la ametrina y el diurón (Morales,1987), los cuales se recomiendan en aplicación postemergente y tienen una residualidad de uno a dos meses, dependiendo de la humedad y el tipo de suelo. Ambos herbicidas pueden no controlar algunas malezas gramíneas que se presentan comúnmente en este cultivo.

El clomazone es un herbicida muy eficiente para el control de malezas gramíneas y algunas especies de hoja ancha (Thomson, 1993). En caña de azúcar puede aplicarse solo en preemergencia (FMC, s/f) o mezclado con otros herbicidas como la ametrina, en postemergencia, con lo que se incrementa el espectro de male-

\footnotetext{
1 Presentado en la XLIV Reunión Anual del PCCMCA, Nicaragua, 1998.

2 Investigador de Maleza y su Control del Campo Experimental Cotaxtla. CIRGOC. INIFAP. SAGAR, México.
} 
zas controladas (Câmara et al, 1995; Christoffoleti et $a l, 1995)$. Experiencias previas han indicado que la aplicación postemergente del clomazone ocasiona un "blanqueamiento" temporal a las hojas de la caña de azúcar, que están expuestas al momento de la aplicación. Sin embargo, se ha demostrado que este "blanqueamiento" no tiene efectos negativos en el rendimiento o calidad de las plantas cosechadas (Orsi y Gallo, 1995).

De acuerdo a las características antes mencionadas, el clomazone, solo o en mezcla con ametrina, puede llegar a representar una alternativa confiable para el control de malezas en las siembras de caña de azúcar del estado de Veracruz.

\section{MATERIALES Y MÉTODOS}

Se establecieron en 1997 dos experimentos en condiciones de riego en la región cañera de la Cuenca Baja del río Papaloapan en el estado de Veracruz, México. Un experimento se estableció en el rancho "Los Piñones", municipio de Tierra Blanca y el otro en el Ejido Porvenir, municipio de Tres Valles. En ambos casos, las parcelas experimentales se sembraron con la cantidad equivalente a $12.000 \mathrm{~kg} / \mathrm{ha}$ de caña de azúcar de la variedad MEX-69-290.

En los dos experimentos se evaluaron los 11 tratamientos que se describen en el Cuadro 1. Los tratamientos fueron distribuídos en el terreno de acuerdo a un diseño experimental de bloques completos al azar con cuatro repeticiones. En el rancho Los Piñones, las parcelas experimentales estuvieron constituídas por cinco surcos de $10 \mathrm{~m}$ de longitud y 1,2 m de separación entre surcos, mientras que en el Ejido Porvenir, éstas fueron de cinco surcos de ocho metros de longitud y 1,3 $\mathrm{m}$ de separación entre surcos.

Los herbicidas se aplicaron con una aspersora motorizada de mochila, equipada con cuatro boquillas Tee jet 8002. El volumen de agua aplicado fue equivalente a 230 1/ha. Los tratamientos del uno al cinco se aplicaron en preemergencia, mientras que los tratamientos del seis al 10, se aplicaron cuando la caña de azúcar tenía entre 30 y $40 \mathrm{~cm}$ de altura y la mayoría de las malezas tenían una altura entre cinco y $15 \mathrm{~cm}$. En todos los casos, solamente se aplicaron los tres surcos centrales de las parcelas, y se permitió el desarrollo de la maleza en los dos surcos laterales de cada parcela, a fin de utilizarlos como testigos 1 enhierbados al momento de las evaluaciones. En las parcelas correspondientes a los testigos enhierbados se permitió el libre desarrollo de la maleza.
Cuadro 1. Descripción de los tratamientos del experimento de evaluación del herbicida clomazone, solo y en mezcla con ametrina en caña de azúcar. Campo Experimental Cotaxtla. CIRGOC. INIFAP. SAGAR. 1997.

\begin{tabular}{llrl}
\hline No. Tratamiento & $\begin{array}{c}\text { Dosis } \\
\text { (g i.a./ha) }\end{array}$ & $\begin{array}{c}\text { Época de } \\
\text { aplicación }\end{array}$ \\
\hline 1 & Clomazone & 960 & Preemergencia \\
2 & Clomazone & 1200 & Preemergencia \\
3 & Clomazone & 1440 & Preemergencia \\
4 & $*$ Diurón+Hexazinona & $409+1091$ & Preemergencia \\
5 & $*$ Ametrina+Atrazina & $1200+1200$ & Preemergencia \\
6 & $*$ Clomazone+Ametrina & $600+900$ & Maleza $5-15 \mathrm{~cm}$ \\
7 & $*$ Clomazone+Ametrina & $800+1200$ & Maleza $5-15 \mathrm{~cm}$ \\
8 & $*$ Clomazone+Ametrina & $1000+1500$ & Maleza $5-15 \mathrm{~cm}$ \\
9 & $* *$ Diurón + 2,4-D & $1600+960$ & Maleza $5-15 \mathrm{~cm}$ \\
10 **Ametrina+2,4-D & $1225+650$ & Maleza $5-15 \mathrm{~cm}$ \\
11 & Testigo enhierbado & & \\
\hline
\end{tabular}

Nota: a los tratamientos del 6 al 10 se les adicionó el surfactante LI700 en dosis de 1,5 ml/litro de agua vertida.

* mezcla de fábrica

** mezcla de tanque

La densidad de población de malezas se determinó a los 15, 30, 60 y 90 días después de la aplicación de los tratamientos (DDA). En cada muestreo se utilizó un cuadrante de $0,5 \times 0,5 \mathrm{~m}$, el cual fue lanzado al azar en dos ocasiones en cada una de las parcelas correspondientes a los testigos enhierbados. Las malezas de los cuadrantes se identificaron y cuantificaron para luego determinar la densidad de población por hectárea.

Las evaluaciones de control de malezas se llevaron a cabo en las mismas fechas en que su densidad de población se cuantificó, esto es, a los 15, 30, 60 y 90 DDA. Se evaluó visualmente el efecto de los herbicidas en las malezas dominantes; para esto se compararon las poblaciones, grados de desarrollo y vigor de las malezas de los surcos laterales no aplicados, con los mismos parámetros de las malezas presentes en los surcos aplicados y se les asignó un valor en la escala de cero a $100 \%$, en donde cero significó que la maleza no fue afectada y $100 \%$ que fue completamente destruída.

Las evaluaciones de toxicidad también se efectuaron a los 15, 30, 60 y 90 DDA. Debido a los síntomas de "blanqueamiento" ocasionados por el clomazone, fue necesario evaluar este tipo de toxicidad por separado de aquella que se presentó en forma de tejido necrótico. En ambos casos se utilizó la escala de cero a $100 \%$, en donde para el caso de necrosis foliar, cero significó que la caña no fue afectada y $100 \%$ que fue completamente destruída. Para el caso de "blanquea- 
miento", cero significó que la caña de azúcar no sufrió ninguna decoloración y $100 \%$ que todo el follaje era de color blanco. Los valores asignados al "blanqueamiento" del follaje se presentan entre paréntesis en los cuadros respectivos.

Para homogenizar las varianzas, los datos experimentales de porcentaje de control de cada una de las malezas se transformaron a un valor de arco seno, de acuerdo a lo que se recomienda por Gómez y Gómez (1984). Los análisis de varianza se efectuaron con los datos transformados y como prueba de separación de medias se utilizó Tukey al 0,05. Aún cuando los análisis de varianza y las pruebas de separación de medias se efectuaron con datos transformados, por motivos de claridad se presentan los datos originales.

\section{RESULTADOS}

\section{Experimento del rancho Los Piñones}

En el sitio experimental, la densidad de población de las malezas fue de 2.305.000 plantas/ha a los 15 DDA y fue disminuyendo hasta cuantificar una densidad de 1.330.000 plantas/ha a los 90 DDA. Las especies de malezas dominantes fueron: Echinochloa colona, Phyllanthus niruri, Cyperus rotundus, Lagascea mollis y Amaranthus spinosus.

A los 15 DDA, las tres dosis de clomazone aplicadas en preemergencia tuvieron un $100 \%$ de control de E. colona. Con la dosis de $1440 \mathrm{~g} / \mathrm{ha}$, el control total se extendió hasta los 90 DDA, mientras que los controles obtenidos con las dosis de 960 y $1200 \mathrm{~g} / \mathrm{ha}$ disminuyerone entre cada época de evaluación, hasta cuantificar a los 90 DDA, niveles del 91,25 y $95,75 \%$, para la dosis baja e intermedia, respectivamente.

Los tratamientos preemergentes de las mezclas de diurón+hexazinona y ametrina+atrazina ejercieron un excelente control inicial de E. colona. Sin embargo, el efecto de la primera mezcla se redujo fuertemente entre los 15 y 30 DDA, por lo que a los 90 DDA el control fue de sólo un $65 \%$. A su vez, en esta misma época de evaluación, el control observado por la mezcla de ametrina+atrazina fue de $87,5 \%$, el cual fue estadísticamente semejante al obtenido con la dosis de $960 \mathrm{~g} / \mathrm{ha}$ de clomazone.

Los controles de E. colona obtenidos con las mezclas de clomazone+ametrina fueron de 86,25; 98,75 y $99,25 \%$ con las dosis altas, intermedias y bajas respectivamente. Con las dosis altas, los controles se mantuvieron alrededor del $95 \%$ a los 90 DDA; con las dosis intermedias el control en la última época de evaluación se redujo a $87,5 \%$, mientras que con las dosis bajas, fue de $55 \%$. La mezcla de 2,4-D+ diurón y de ametrina+2,4-D mostraron controles iniciales de esta especie del 42,5 y $70 \%$, respectivamente, aunque el efecto se perdió entre los 30 y 60 DDA con ametrina+2,4-D y entre los 60 y 90 DDA con la mezcla de diurón + 2,4-D (Cuadro 2).

Cuadro 2. Efecto de los tratamientos en el control de Echinochloa colona a los 15, 30, 60 y 90 días después de su aplicación. Campo Experimental Cotaxtla. CIRGOC. INIFAP. SAGAR. 1997.

\begin{tabular}{ccccc}
\hline Trat. & $\mathbf{1 5}$ & $\mathbf{3 0}$ & $\mathbf{6 0}$ & $\mathbf{9 0}$ \\
\hline 1 & $100 \mathrm{a}$ & $93,75 \mathrm{bcd}$ & $91,25 \mathrm{bc}$ & $91,25 \mathrm{bc}$ \\
2 & $100 \mathrm{a}$ & $99,75 \mathrm{ab}$ & $95,75 \mathrm{ab}$ & $95,75 \mathrm{~b}$ \\
3 & $100 \mathrm{a}$ & $100 \mathrm{a}$ & $100 \mathrm{a}$ & $100 \mathrm{a}$ \\
4 & $99 \mathrm{a}$ & $77,5 \mathrm{e}$ & $77,5 \mathrm{~cd}$ & $65 \mathrm{~d}$ \\
5 & $100 \mathrm{a}$ & $100 \mathrm{a}$ & $95,75 \mathrm{ab}$ & $87,5 \mathrm{c}$ \\
6 & $86,25 \mathrm{~b}$ & $82,5 \mathrm{de}$ & $65 \mathrm{~d}$ & $55 \mathrm{~d}$ \\
7 & $98,75 \mathrm{a}$ & $91,25 \mathrm{cde}$ & $90 \mathrm{bc}$ & $87,5 \mathrm{c}$ \\
8 & $99,25 \mathrm{a}$ & $97,75 \mathrm{abc}$ & $94,5 \mathrm{abc}$ & $94,5 \mathrm{~b}$ \\
9 & $42,5 \mathrm{~d}$ & $42,5 \mathrm{f}$ & $12,5 \mathrm{e}$ & $0 \mathrm{e}$ \\
10 & $70 \mathrm{c}$ & $40 \mathrm{f}$ & $0 \mathrm{f}$ & $0 \mathrm{e}$ \\
11 & $0 \mathrm{e}$ & $0 \mathrm{~g}$ & $0 \mathrm{f}$ & $0 \mathrm{e}$ \\
\hline
\end{tabular}

Las letras a la derecha de los valores representan la prueba de Tukey $(0,05)$. Valores con la misma letra no son significativamente diferentes entre sí. Las comparaciones son entre tratamientos en cada una de las épocas de evaluación.

Todos los tratamientos químicos tuvieron un 100\% de control de P. niruri a los 15 DDA. El control total de esta maleza se mantuvo hasta los 90 DDA, con las mezclas preemergentes de diurón+hexazinona y ametrina+atrazina y la dosis más alta de la mezcla de clomazone+ametrina aplicada en postemergencia. Los tratamientos con las dosis intermedias y bajas de esta mezcla tuvieron un control de $P$. niruri de 93,75 y $85 \%$, respectivamente. El efecto del clomazone aplicado en preemergencia se redujo fuertemente entre los 60 y 90 DDA, sobre todo con las dosis de 960 y $1200 \mathrm{~g} / \mathrm{ha}$, con las cuales se controló un 45 y $60 \%$, respectivamente. El control de P. niruri con diurón + 2,4-D fue del $80 \%$, mientras que con ametrina+2,4-D se controló el $71,25 \%$ (Cuadro 3).

Ninguno de los tratamientos aplicados en preemergencia tuvo efecto sobre $C$. rotundus. De los tratamientos postemergentes, sólo diurón+2,4-D controló esta maleza a los 15 y 30 DDA. Sin embargo, a partir de los 60 DDA su efecto se perdió completamente. Clomazone+ametrina y amterina+2,4-D brindaron un control 
Cuadro 3. Efecto de los tratamientos en el control de Phyllanthus niruri a los 15, 30, 60 y 90 días después de su aplicación. Campo Experimental Cotaxtla. CIRGOC. INIFAP. SAGAR. 1997.

\begin{tabular}{ccccc}
\hline Trat. & $\mathbf{1 5}$ & $\mathbf{3 0}$ & $\mathbf{6 0}$ & $\mathbf{9 0}$ \\
\hline 1 & $100 \mathrm{a}$ & $88,75 \mathrm{~d}$ & $77,5 \mathrm{de}$ & $45 \mathrm{e}$ \\
2 & $100 \mathrm{a}$ & $94,5 \mathrm{bcd}$ & $83,75 \mathrm{de}$ & $60 \mathrm{de}$ \\
3 & $100 \mathrm{a}$ & $97,5 \mathrm{ab}$ & $95 \mathrm{abc}$ & $83,75 \mathrm{bc}$ \\
4 & $100 \mathrm{a}$ & $100 \mathrm{a}$ & $100 \mathrm{a}$ & $100 \mathrm{a}$ \\
5 & $100 \mathrm{a}$ & $100 \mathrm{a}$ & $100 \mathrm{a}$ & $100 \mathrm{a}$ \\
6 & $100 \mathrm{a}$ & $97,5 \mathrm{abc}$ & $91,25 \mathrm{bcd}$ & $85 \mathrm{bc}$ \\
7 & $100 \mathrm{a}$ & $99,5 \mathrm{a}$ & $97 \mathrm{ab}$ & $93,75 \mathrm{~b}$ \\
8 & $100 \mathrm{a}$ & $100 \mathrm{a}$ & $100 \mathrm{a}$ & $100 \mathrm{a}$ \\
9 & $100 \mathrm{a}$ & $92,5 \mathrm{~cd}$ & $87,5 \mathrm{cde}$ & $80 \mathrm{c}$ \\
10 & $100 \mathrm{a}$ & $100 \mathrm{a}$ & $75 \mathrm{e}$ & $71,25 \mathrm{~cd}$ \\
11 & $0 \mathrm{~b}$ & $0 \mathrm{e}$ & $0 \mathrm{f}$ & $0 \mathrm{f}$ \\
\hline
\end{tabular}

Las letras a la derecha de los valores representan la prueba de Tukey $(0,05)$. Valores con la misma letra no son significativamente diferentes entre sí. Las comparaciones son entre tratamientos en cada una de las épocas de evaluación.

inicial en $C$. rotundus, pero este desapareció rápidamente (Cuadro 4).

El control sobre L. mollis fue del $100 \%$ hasta los 90 DDA con las dosis más alta de clomazone, ametrina+atrazina en preemergencia y con clomazone+ametrina en postemergencia. A los $90 \mathrm{DDA}$, el resto de los tratamientos controlaron al $90 \%$ de esta especie, con excepción de diurón+2,4-D y ametrina+2,4-D, donde el control fue del 80 y $85 \%$ en postemergencia (Cuadro 5).

Cuadro 4. Efecto de los tratamientos en el control de $C y$ perus rotundus a los 15, 30, 60 y 90 días después de su aplicación. Campo Experimental Cotaxtla. CIRGOC. INIFAP. SAGAR. 1997.

\begin{tabular}{lllll}
\hline $\begin{array}{l}\text { Trata- } \\
\text { mientos }\end{array}$ & $\mathbf{5}$ & \multicolumn{3}{c}{ Días } \\
\cline { 2 - 5 } & $\mathbf{1 5}$ & $\mathbf{3 0}$ & $\mathbf{6 0}$ & $\mathbf{9 0}$ \\
\hline 1 & $0 \mathrm{c}$ & $0 \mathrm{c}$ & 0 & 0 \\
2 & $0 \mathrm{c}$ & $0 \mathrm{c}$ & 0 & 0 \\
3 & $0 \mathrm{c}$ & $0 \mathrm{c}$ & 0 & 0 \\
4 & $0 \mathrm{c}$ & $0 \mathrm{c}$ & 0 & 0 \\
5 & $0 \mathrm{c}$ & $0 \mathrm{c}$ & 0 & 0 \\
6 & $13,75 \mathrm{bc}$ & $0 \mathrm{c}$ & 0 & 0 \\
7 & $15 \mathrm{bc}$ & $0 \mathrm{c}$ & 0 & 0 \\
8 & $18,75 \mathrm{bc}$ & $0 \mathrm{c}$ & 0 & 0 \\
9 & $85 \mathrm{a}$ & $62,5 \mathrm{a}$ & 0 & 0 \\
10 & $32,5 \mathrm{~b}$ & $17,5 \mathrm{~b}$ & 0 & 0 \\
11 & $0 \mathrm{c}$ & $0 \mathrm{c}$ & 0 & 0 \\
\hline
\end{tabular}

Las letras a la derecha de los valores representan la prueba de Tukey $(0,05)$. Valores con la misma letra no son significativamente diferentes entre sí. Las comparaciones son entre tratamientos en cada una de las épocas de evaluación.
Cuadro 5. Efecto de los tratamientos en el control de $\mathrm{La}$ gascea mollis a los 15, 30, 60 y 90 días después de su aplicación. Campo Experimental Cotaxtla. CIRGOC. INIFAP. SAGAR. 1997.

\begin{tabular}{cccll}
\hline Tratam. & $\mathbf{1 5}$ & $\mathbf{3 0}$ & $\mathbf{6 0}$ & \multicolumn{1}{c}{$\mathbf{9 0}$} \\
\hline 1 & $100 \mathrm{a}$ & $100 \mathrm{a}$ & $100 \mathrm{a}$ & $94,5 \mathrm{abc}$ \\
2 & $100 \mathrm{a}$ & $100 \mathrm{a}$ & $100 \mathrm{a}$ & $91,25 \mathrm{bc}$ \\
3 & $100 \mathrm{a}$ & $100 \mathrm{a}$ & $100 \mathrm{a}$ & $100 \mathrm{a}$ \\
4 & $100 \mathrm{a}$ & $100 \mathrm{a}$ & $100 \mathrm{a}$ & $93,75 \mathrm{abc}$ \\
5 & $100 \mathrm{a}$ & $100 \mathrm{a}$ & $100 \mathrm{a}$ & $100 \mathrm{a}$ \\
6 & $97,75 \mathrm{~b}$ & $94,5 \mathrm{~b}$ & $93,25 \mathrm{~b}$ & $91,25 \mathrm{bc}$ \\
7 & $100 \mathrm{a}$ & $100 \mathrm{a}$ & $100 \mathrm{a}$ & $99 \mathrm{ab}$ \\
8 & $100 \mathrm{a}$ & $100 \mathrm{a}$ & $100 \mathrm{a}$ & $100 \mathrm{a}$ \\
9 & $100 \mathrm{a}$ & $98,75 \mathrm{ab}$ & $90 \mathrm{bc}$ & $83,75 \mathrm{c}$ \\
10 & $100 \mathrm{a}$ & $96,25 \mathrm{ab}$ & $85 \mathrm{c}$ & $80 \mathrm{c}$ \\
11 & $0 \mathrm{c}$ & $0 \mathrm{c}$ & $0 \mathrm{~d}$ & $0 \mathrm{~d}$
\end{tabular}

Las letras a la derecha de los valores representan la prueba de Tukey $(0,05)$. Valores con la misma letra no son significativamente diferentes entre sí. Las comparaciones son entre tratamientos en cada una de las épocas de evaluación.

Los tratamientos de clomazone en preemergencia no controlaron A. spinosus. Con el resto de los tratamientos preemergentes y postemergentes se tuvo un control del 95 a $100 \%$ hasta los 30 DDA, y de 92,5 a $100 \%$ a los 90 DDA (Cuadro 6).

Ninguno de los tratamientos en preemergencia ocasionaron síntomas de toxicidad en las plantas de caña. Por su parte, todos los tratamientos en postemergencia ocasionaron pequeñas áreas necróticas en la

Cuadro 6. Efecto de los tratamientos en el control de Amaranthus spinosus a los 15, 30, 60 y 90 días después de su aplicación. Campo Experimental Cotaxtla. CIRGOC. INIFAP. SAGAR. 1997.

\begin{tabular}{crrrr}
\hline Tratam. & $\mathbf{1 5}$ & $\mathbf{3 0}$ & $\mathbf{6 0}$ & $\mathbf{9 0}$ \\
\hline 1 & $0 \mathrm{c}$ & $0 \mathrm{c}$ & $0 \mathrm{c}$ & $0 \mathrm{c}$ \\
2 & $0 \mathrm{c}$ & $0 \mathrm{c}$ & $0 \mathrm{c}$ & $0 \mathrm{c}$ \\
3 & $0 \mathrm{c}$ & $0 \mathrm{c}$ & $0 \mathrm{c}$ & $0 \mathrm{c}$ \\
4 & $98 \mathrm{~b}$ & $95 \mathrm{~b}$ & $92,5 \mathrm{~b}$ & $92,5 \mathrm{~b}$ \\
5 & $100 \mathrm{a}$ & $100 \mathrm{a}$ & $100 \mathrm{a}$ & $100 \mathrm{a}$ \\
6 & $100 \mathrm{a}$ & $100 \mathrm{a}$ & $100 \mathrm{a}$ & $100 \mathrm{a}$ \\
7 & $100 \mathrm{a}$ & $100 \mathrm{a}$ & $100 \mathrm{a}$ & $100 \mathrm{a}$ \\
8 & $100 \mathrm{a}$ & $100 \mathrm{a}$ & $100 \mathrm{a}$ & $100 \mathrm{a}$ \\
9 & $100 \mathrm{a}$ & $100 \mathrm{a}$ & $95 \mathrm{~b}$ & $92,5 \mathrm{~b}$ \\
10 & $100 \mathrm{a}$ & $100 \mathrm{a}$ & $100 \mathrm{a}$ & $100 \mathrm{a}$ \\
11 & $0 \mathrm{c}$ & $0 \mathrm{c}$ & $0 \mathrm{c}$ & $0 \mathrm{c}$ \\
\hline
\end{tabular}

Las letras a la derecha de los valores representan la prueba de Tukey $(0,05)$. Valores con la misma letra no son significativamente diferentes entre sí. Las comparaciones son entre tratamientos en cada una de las épocas de evaluación. 
punta o los bordes de las hojas al momento de la aplicación. A los 15 DDA, el valor máximo cuantificado fue de $11,75 \%$ con la dosis más alta de clomazone+ametrina. Sin embargo, las áreas necróticas desaparecieron entre los 15 y 30 DDA.

Con clomazone+ametrina en postemergencia, se observó un "blanqueamiento" parcial en el follaje de la caña. El área donde se observó dicho efecto varió entre $21,25 \%$ con las dosis más bajas y $38.75 \%$ con las dosis más altas de esa mezcla y disminuyó significativamente entre los 15 y 30 DDA, y desapareció entre los 30 y 60 DDA (Cuadro 7).

\section{Experimento del Ejido Porvenir}

En esta localidad, la densidad de malezas fue de 2.135.000 plantas/ha a los 15 DDA y disminuyó entre un muestreo y el siguiente, hasta cuantificar 1.080.000 plantas/ha a los 90 DDA. Las especies de malezas dominantes fueron: Phyllanthus niruri, Echinochloa colona, Rottboellia cochinchinensis, Panicum fasciculatum, Mollugo verticillata y Croton lobatus.

A los 15 días de aplicados los tratamientos preemergentes, prácticamente no existía maleza emergida. Por esta razón, en los Cuadros del 8 al 14, no existen valores de control de malezas para los tratamientos uno al cinco.

Todos los tratamientos en postemergencia mostraron un control total de $P$. niruri desde los 15 hasta los 90 DDA. De los tratamientos preemergentes, también

Cuadro 7. Efecto de los tratamientos en la toxicidad a la caña de azúcar (\%) con base en tejido necrótico a los $15,30,60$ y 90 días después de su aplicación. Campo Experimental Cotaxtla. CIRGOC. INIFAP. SAGAR. 1997.

\begin{tabular}{crrrr}
\hline Tratam. & 15 & 30 & $\mathbf{6 0}$ & $\mathbf{9 0}$ \\
\hline 1 & - & 0 & 0 & 0 \\
2 & - & 0 & 0 & 0 \\
3 & - & 0 & 0 & 0 \\
4 & - & 0 & 0 & 0 \\
5 & - & 0 & 0 & 0 \\
6 & $7,5(21,25)$ & $0(5,5)$ & $0(0)$ & $0(0)$ \\
7 & $8,25(27,5)$ & $0(8,5)$ & $0(0)$ & $0(0)$ \\
8 & $11,75(38,75)$ & $0(8,75)$ & $0(0)$ & $0(0)$ \\
9 & 10.5 & 0 & 0 & 0 \\
10 & 6.25 & 0 & 0 & 0 \\
11 & 0 & 0 & 0 & 0 \\
\hline
\end{tabular}

Valores en paréntesis representan el porcentaje de toxicidad con base en blanqueamiento de follaje. Para tatamiento del uno al cinco no aparecen valores ya que no había emergido la caña. clomazone en dosis de 1200 y $1440 \mathrm{~g} / \mathrm{ha}$ y ametrina+atrazina controlaron el $100 \%$ hasta los 30 DDA. A su vez, a los 90 DDA, sólo el último tratamiento mostró controles de $P$. niruri estadísticamente semejantes a los porcentajes obtenidos con los tratamientos postemergentes, mientras que en el resto de éstos, los controles se redujeron significativamente entre 45 y $73,75 \%$ (Cuadro 8).

A los 30 DDA, los mejores controles de E. colona con los tratamientos en preemergencia se tuvieron con clomazone en dosis de 1200 y $1440 \mathrm{~g} / \mathrm{ha}$, en ambos casos superiores al 95\%. La dosis de $960 \mathrm{~g} / \mathrm{ha}$ de clomazone y diurónthexazinona controron alrededor del $80 \%$. Mientras que el control con ametrina+atrazina fue de sólo 55\%. A los 90 DDA, entre los tratamientos preemergentes, solamente las dosis alta e intermedia de clomazone mantenían controles superiores al $90 \%$.

De los tratamientos postemergentes, a los 15 DDA, las dosis intermedia y alta de clomazone+ametrina y ametrina+2,4-D controlaron E. colona en un 87,5 a $95 \%$. A su vez, en esa misma época, con la dosis baja de clomazone+ametrina y con diurón $+2,4-\mathrm{D}$, los controles obtenidos fueron de un 75\%. A los 90 DDA, solo con la dosis de $1000+1500 \mathrm{~g} / \mathrm{ha}$ de clomazone+ ametrina se obtuvieron controles estadísticamente semejantes a los obtenidos con las dosis intermedia y alta de clomazone en preemergencia (Cuadro 9).

A los 30 DDA, los mejores controles de $R$. cochinchinensis con los tratamientos preemergentes se obtuvieron con las dosis de 1200 y $1440 \mathrm{~g} / \mathrm{ha}$ de clomazone,

Cuadro 8. Efecto de los tratamientos en el control de Phyllanthus niruri a los 15, 30, 60 y 90 días después de su aplicación. Campo Experimental Cotaxtla. CIRGOC. INIFAP. SAGAR. 1997.

\begin{tabular}{crrrr}
\hline Tratam. & $\mathbf{1 5}$ & \multicolumn{1}{c}{$\mathbf{3 0}$} & $\mathbf{6 0}$ & \multicolumn{1}{c}{$\mathbf{9 0}$} \\
\hline 1 & - & $72.5 \mathrm{~b}$ & $56.25 \mathrm{~d}$ & $45 \mathrm{c}$ \\
2 & - & $100 \mathrm{a}$ & $82.5 \mathrm{bc}$ & $75 \mathrm{~b}$ \\
3 & - & $100 \mathrm{a}$ & $87.5 \mathrm{~b}$ & $73.75 \mathrm{~b}$ \\
4 & - & $72.5 \mathrm{~b}$ & $70 \mathrm{~cd}$ & $63.75 \mathrm{bc}$ \\
5 & - & $100 \mathrm{a}$ & $100 \mathrm{a}$ & $96.25 \mathrm{a}$ \\
6 & 100 & $100 \mathrm{a}$ & $100 \mathrm{a}$ & $100 \mathrm{a}$ \\
7 & 100 & $100 \mathrm{a}$ & $100 \mathrm{a}$ & $100 \mathrm{a}$ \\
8 & 100 & $100 \mathrm{a}$ & $100 \mathrm{a}$ & $100 \mathrm{a}$ \\
9 & 100 & $100 \mathrm{a}$ & $100 \mathrm{a}$ & $100 \mathrm{a}$ \\
10 & 100 & $100 \mathrm{a}$ & $100 \mathrm{a}$ & $100 \mathrm{a}$ \\
11 & 0 & $0 \mathrm{c}$ & $0 \mathrm{e}$ & $0 \mathrm{~d}$ \\
\hline
\end{tabular}

Las letras a la derecha de los valores representan la prueba de Tukey $(0,05)$. Valores con la misma letra no son significativamente diferentes entre sí. Las comparaciones son entre tratamientos en cada una de las épocas de evaluación. 
Cuadro 9. Efecto de los tratamientos en el control de Echinochloa colona a los 15, 30, 60 y 90 días después de su aplicación Campo Experimental Cotaxtla. CIRGOC. INIFAP. SAGAR. 1997.

\begin{tabular}{cclll}
\hline Trat. & $\mathbf{1 5}$ & \multicolumn{1}{c}{$\mathbf{3 0}$} & $\mathbf{6 0}$ & \multicolumn{1}{c}{$\mathbf{9 0}$} \\
\hline 1 & - & $80 \mathrm{bcd}$ & $76,25 \mathrm{c}$ & $72,5 \mathrm{~cd}$ \\
2 & - & $95,25 \mathrm{ab}$ & $94.5 \mathrm{a}$ & $91,25 \mathrm{ab}$ \\
3 & - & $97,75 \mathrm{a}$ & $93,75 \mathrm{ab}$ & $92,5 \mathrm{a}$ \\
4 & - & $81,25 \mathrm{bcd}$ & $76,25 \mathrm{bc}$ & $73,75 \mathrm{c}$ \\
5 & - & $55 \mathrm{e}$ & $40 \mathrm{e}$ & $27,5 \mathrm{~d}$ \\
6 & $76,25 \mathrm{bc}$ & $76,25 \mathrm{cde}$ & $65 \mathrm{~cd}$ & $55 \mathrm{~d}$ \\
7 & $87.5 \mathrm{ab}$ & $80 \mathrm{bcd}$ & $77,5 \mathrm{bc}$ & $72,5 \mathrm{~cd}$ \\
8 & $91 \mathrm{a}$ & $87,5 \mathrm{abc}$ & $83,75 \mathrm{abc}$ & $80 \mathrm{bc}$ \\
9 & $75 \mathrm{c}$ & $60 \mathrm{de}$ & $45 \mathrm{de}$ & $27,5 \mathrm{e}$ \\
10 & $95 \mathrm{a}$ & $93,25 \mathrm{ab}$ & $82,5 \mathrm{abc}$ & $73,75 \mathrm{c}$ \\
11 & $0 \mathrm{~d}$ & $0 \mathrm{f}$ & $0 \mathrm{f}$ & $0 \mathrm{f}$ \\
\hline
\end{tabular}

Las letras a la derecha de los valores representan la prueba de Tukey $(0,01)$. Valores con la misma letra no son significativamente diferentes entre sí. Las comparaciones son entre tratamientos en cada una de las épocas de evaluación.

donde el control fue de un 94,5 y $96,5 \%$, respectivamente. Ambos tratamientos mantuvieron controles superiores al $90 \%$ hasta los 60 DDA y a los 90 DDA eran de un 87,5 y $90 \%$.

La mezcla de diurón+hexazinona controló $R$. $c o$ chinchinensis en un $80 \%$ a los 30 DDA, pero a los 90 DDA fue de sólo el 65\%. Ametrina+atrazina, controló menos del 50\% desde los 30 DDA.

Con los tratamientos postemergentes, a los 15 DDA, ametrina+2,4-D mostró los controles más altos de $R$. cochinchinensis con $94,5 \%$, seguido por clomazone+ametrina a $1000+1500 \mathrm{~g} / \mathrm{ha}$, con $87,5 \%$. A los 90 DDA, el control de $R$. cochinchinensis con estos tratamientos se redujo a 72,5 y $75 \%$, respectivamente. Los controles obtenidos con los otros tratamientos en postemergencia variaron del 55 al $75 \%$ a los 15 DDA y del 7,5 al $67,5 \%$ a los 90 DDA (Cuadro 10).

A los 30 DDA, las tres dosis de clomazone aplicadas en preemergencia, mostraban controles de $P$. fasciculatum de 90 a $100 \%$. Los controles se mantuvieron por arriba del $90 \%$ a los 60 y 90 DDA con las dosis de 1200 y $1440 \mathrm{~g} / \mathrm{ha}$, mientras que con la dosis de $960 \mathrm{~g} / \mathrm{ha}$ el control en la última época de evaluación fue de $82.5 \%$.

Diurón+hexazinona y ametrina+atrazina controlaron $P$. fasciculatum en un 83,75 y $71,25 \%$, respectivamente a los 30 DDA, los cuales se redujeron a 62,5 y $42,5 \%$ a los 90 DDA.
Cuadro 10. Efecto de los tratamientos en el control de Rottboellia cochinchinensis a los 15, 30, 60 y 90 días después de su aplicación. Campo Experimental Cotaxtla. CIRGOC. INIFAP. SAGAR. 1997.

\begin{tabular}{cclll}
\hline Trat. & $\mathbf{1 5}$ & $\mathbf{3 0}$ & $\mathbf{6 0}$ & $\mathbf{9 0}$ \\
\hline 1 & - & $78,75 \mathrm{bc}$ & $73,75 \mathrm{ab}$ & $71,25 \mathrm{ab}$ \\
2 & - & $94,5 \mathrm{ab}$ & $91,25 \mathrm{a}$ & $87,5 \mathrm{ab}$ \\
3 & - & $96,5 \mathrm{a}$ & $92,5 \mathrm{a}$ & $90 \mathrm{a}$ \\
4 & - & $80 \mathrm{bc}$ & $68,75 \mathrm{ab}$ & $65 \mathrm{bc}$ \\
5 & - & $45 \mathrm{de}$ & $32,5 \mathrm{c}$ & $17,5 \mathrm{de}$ \\
6 & $55 \mathrm{~d}$ & $52,5 \mathrm{de}$ & $50 \mathrm{bc}$ & $40 \mathrm{~cd}$ \\
7 & $75 \mathrm{bc}$ & $71,25 \mathrm{~cd}$ & $70 \mathrm{ab}$ & $67,5 \mathrm{ab}$ \\
8 & $87,5 \mathrm{ab}$ & $87,5 \mathrm{abc}$ & $81,25 \mathrm{ab}$ & $75 \mathrm{ab}$ \\
9 & $62,5 \mathrm{~cd}$ & $40 \mathrm{e}$ & $27,5 \mathrm{c}$ & $7,5 \mathrm{ef}$ \\
10 & $94,5 \mathrm{a}$ & $90 \mathrm{abc}$ & $78,75 \mathrm{ab}$ & $72,5 \mathrm{ab}$ \\
11 & $0 \mathrm{e}$ & $0 \mathrm{f}$ & $0 \mathrm{~d}$ & $0 \mathrm{f}$ \\
\hline
\end{tabular}

Las letras a la derecha de los valores representan la prueba de Tukey $(0,05)$. Valores con la misma letra no son significativamente diferentes entre sí. Las comparaciones son entre tratamientos en cada una de las épocas de evaluación.

Con las aplicaciones postemergentes, a los 15 DDA, todos los tratamientos controlaron P. fasciculatum en más del 90\%, a excepción del diurón+2,4-D, cuyo control fue de $80 \%$. A los 60 DDA, solamente la dosis de $1000+1500 \mathrm{~g} /$ ha de clomazone+ametrina brindó controles superiores al $90 \%$ de dicha especie y a los 90 DDA, los mayores controles (en postemergencia) se obtuvieron con las dosis de $800+1200$ y $1000+1500$ $\mathrm{g} /$ ha de clomazone+ametrina, con 82,5 y $85 \%$, respectivamente (Cuadro 11).

Cuadro 11. Efecto de los tratamientos en el control de Panicum fasciculatum a los 15, 30, 60 y 90 días después de su aplicación. Campo Experimental Cotaxtla. CIRGOC. INIFAP. SAGAR. 1997.

\begin{tabular}{cllll}
\hline Tratam. & $\mathbf{1 5}$ & $\mathbf{3 0}$ & $\mathbf{6 0}$ & \multicolumn{1}{c}{$\mathbf{9 0}$} \\
\hline 1 & - & $90 \mathrm{~cd}$ & $86,25 \mathrm{~cd}$ & $82,5 \mathrm{bc}$ \\
2 & - & $97,75 \mathrm{ab}$ & $96,25 \mathrm{ab}$ & $92,5 \mathrm{ab}$ \\
3 & - & $100 \mathrm{a}$ & $98,25 \mathrm{a}$ & $93,75 \mathrm{a}$ \\
4 & - & $83,75 \mathrm{de}$ & $73,75 \mathrm{de}$ & $62,5 \mathrm{~d}$ \\
5 & - & $71,25 \mathrm{e}$ & $57,5 \mathrm{ef}$ & $42,5 \mathrm{e}$ \\
6 & $91,25 \mathrm{a}$ & $88,75 \mathrm{~d}$ & $82,5 \mathrm{~cd}$ & $73,75 \mathrm{~cd}$ \\
7 & $97 \mathrm{a}$ & $93,75 \mathrm{bcd}$ & $86,25 \mathrm{~cd}$ & $82,5 \mathrm{bc}$ \\
8 & $98,25 \mathrm{a}$ & $97 \mathrm{abc}$ & $91,25 \mathrm{bc}$ & $85, \mathrm{abc}$ \\
9 & $80 \mathrm{~b}$ & $68,75 \mathrm{e}$ & $50 \mathrm{f}$ & $37,5 \mathrm{e}$ \\
10 & $94,5 \mathrm{a}$ & $87,5 \mathrm{~d}$ & $77,5 \mathrm{~d}$ & $72,5 \mathrm{~cd}$ \\
11 & $0 \mathrm{c}$ & $0 \mathrm{f}$ & $0 \mathrm{~g}$ & $0 \mathrm{f}$ \\
\hline
\end{tabular}

Las letras a la derecha de los valores representan la prueba de Tukey $(0,05)$. Valores con la misma letra no son significativamente diferentes entre sí. Las comparaciones son entre tratamientos en cada una de las épocas de evaluación. 
M. verticillata se controló completamente hasta los 90 DDA por diurón+hexazinona y ametrina+atrazina en preemergencia y todos los tratamientos en postemergencia. Por su parte, las dosis de 1200 y $1400 \mathrm{~g}$ de clomazone/ha ejercieron un $\mathrm{m}$ efecto inicial muy ligeron sobre esta maleza, pero este desapareció en su totalidad a partir de los 60 DDA (Cuadro 12).

Cuadro 12. Efecto de los tratamientos en el control de $\mathrm{Mo}$ llugo verticillata a los 15, 30, 60 y 90 días después de su aplicación. Campo Experimental Cotaxtla. CIRGOC. INIFAP. SAGAR. 1997.

\begin{tabular}{crrrr}
\hline Tratam. & $\mathbf{1 5}$ & $\mathbf{3 0}$ & $\mathbf{6 0}$ & $\mathbf{9 0}$ \\
\hline 1 & - & $0 \mathrm{~d}$ & 0 & 0 \\
2 & - & $10 \mathrm{c}$ & 0 & 0 \\
3 & - & $17.5 \mathrm{~b}$ & 0 & 0 \\
4 & - & $100 \mathrm{a}$ & 100 & 100 \\
5 & - & $100 \mathrm{a}$ & 100 & 100 \\
6 & 100 & $100 \mathrm{a}$ & 100 & 100 \\
7 & 100 & $100 \mathrm{a}$ & 100 & 100 \\
8 & 100 & $100 \mathrm{a}$ & 100 & 100 \\
9 & 100 & $100 \mathrm{a}$ & 100 & 100 \\
10 & 100 & $100 \mathrm{a}$ & 100 & 100 \\
11 & 0 & $0 \mathrm{~d}$ & 0 & 0 \\
\hline
\end{tabular}

Las letras a la derecha de los valores representan la prueba de Tukey $(0,05)$. Valores con la misma letra no son significativamente diferentes entre sí. Las comparaciones son entre tratamientos en cada una de las épocas de evaluación.

Los tratamientos de clomazone aplicados en preemergencia no brindaron ningún efecto sobre C. lobatus. A su vez, los controles obtenidos en preemergencia con diurón+hexazinona y ametrina+atrazina fueron de 72,5 y $77,5 \%$ respectivamente a los 30 DDA y de 55 y $60 \%$ a los 90 DDA, estos valores de control fueron significativamente menores que en postemergencia en las dos últimas épocas de evaluación.

A los 15 DDA, el control de C. lobatus con los tres tratamientos de clomazone+ametrina fue de $100 \%$, mientras que los controles fueron casi totales con diurón+2,4-D y ametrina+2,4-D. A los 30 DDA, con la dosis de $600+900 \mathrm{~g} / \mathrm{ha}$ de clomazone+ametrina se observó una reducción marcada, a diferencia del resto de los tratamientos postemergentes. Sin embargo, a los 60 y 90 DDA, todos los tratamientos en postemergencia brindaron controles de $C$. lobatus estadísticamente semejantes, oscilando entre 78,75 y 88,75\% (Cuadro 13).

Sólo se observaron síntomas de toxicidad en las plantas de caña, a las que se les aplicó clomazone+ametrina en postermergencia. Se distinguieron dos cla-
Cuadro 13. Efecto de los tratamientos en el control de Croton lobatus a los 15, 30, 60 y 90 días después de su aplicación. Campo Experimental Cotaxtla. CIRGOC. INIFAP. SAGAR. 1997.

\begin{tabular}{crrrr}
\hline Tratam. & \multicolumn{1}{c}{15} & \multicolumn{1}{c}{$\mathbf{3 0}$} & \multicolumn{1}{c}{$\mathbf{0 0}$} & \multicolumn{1}{c}{$\mathbf{9 0}$} \\
\hline 1 & - & $0 \mathrm{e}$ & $0 \mathrm{c}$ & $0 \mathrm{c}$ \\
2 & - & $0 \mathrm{e}$ & $0 \mathrm{c}$ & $0 \mathrm{c}$ \\
3 & - & $0 \mathrm{e}$ & $0 \mathrm{c}$ & $0 \mathrm{c}$ \\
4 & - & $72,5 \mathrm{~d}$ & $57,5 \mathrm{~b}$ & $55 \mathrm{~b}$ \\
5 & - & $77,5 \mathrm{~cd}$ & $60 \mathrm{~b}$ & $60 \mathrm{~b}$ \\
6 & $100 \mathrm{a}$ & $82,5 \mathrm{bcd}$ & $80 \mathrm{a}$ & $78,75 \mathrm{a}$ \\
7 & $100 \mathrm{a}$ & $91,25 \mathrm{ab}$ & $85 \mathrm{a}$ & $82,5 \mathrm{a}$ \\
8 & $100 \mathrm{a}$ & $95,25 \mathrm{a}$ & $85 \mathrm{a}$ & $83,75 \mathrm{a}$ \\
9 & $97,5 \mathrm{a}$ & $94,5 \mathrm{ab}$ & $90 \mathrm{a}$ & $88,75 \mathrm{a}$ \\
10 & $98,25 \mathrm{a}$ & $88,75 \mathrm{abc}$ & $86,25 \mathrm{a}$ & $78,75 \mathrm{a}$ \\
11 & $0 \mathrm{~b}$ & $0 \mathrm{e}$ & $0 \mathrm{c}$ & $0 \mathrm{c}$ \\
\hline
\end{tabular}

Las letras a la derecha de los valores representan la prueba de Tukey $(0,05)$. Valores con la misma letra no son significativamente diferentes entre sí. Las comparaciones son entre tratamientos en cada una de las épocas de evaluación.

ses de síntomas: el primero consistió en pequeñas áreas necróticas localizadas en las puntas y/o los bordes de las hojas, que a los 15 DDA llegaron a ocupar entre el 3,75 y $8 \%$ del área foliar, pero que a los 30 DDA habían desaparecido por completo. El segundo síntoma de toxicidad, consistió en el "blanqueamiento" de algunas áreas del follaje que estaban expuestas al momento de la aplicación. Se observó una correlación directamente proporcional entre la dosis de clomazone+ametrina y el área con "blanqueamiento", de tal forma que a mayor dosis aplicada de este herbicida, se tuvo mayor área foliar afectada. Las mayores áreas con "blanqueamiento" se observaron a los 15 DDA. Estos fueron de 13,75; 16,25 y $21,25 \%$, para las dosis baja, intermedia y alta de la mezcla de clomazone+ametrina. En todos los casos, a los 30 DDA, el área con blanqueamiento se redujo entre 5,5 y $9 \%$ y a partir de los 60 DDA no se observó ningún síntoma de toxicidad (Cuadro 14).

\section{CONCLUSIONES}

El clomazone aplicado en preemergencia tuvo un control de L. mollis superior al $90 \%$ desde $960 \mathrm{~g} / \mathrm{ha}$. Para E.colona la dosis requerida para obtener el $90 \%$ de control varió entre 960 y $1200 \mathrm{~g} / \mathrm{ha}$, esta última dosis fue necesaria para el control de $P$. fasciculatum. A su vez, se necesitaron $1440 \mathrm{~g} /$ ha para controlar entre un 80 y $90 \%$ de $P$. niruri y $R$. cochinchinenesis y no tuvo efecto alguno sobre $C$. rotundus, $M$. verticillata, $C$. lobatus y A. spinosus. 
Cuadro 14. Efecto de los tratamientos en la toxicidad de la caña de azúcar (\%) a los 15, 30, 60 y 90 días después de su aplicación. Campo Experimental Cotaxtla. CIRGOC. INIFAP. SAGAR. 1997.

\begin{tabular}{|c|c|c|c|c|}
\hline Tratam & 15 & 30 & 60 & 90 \\
\hline 1 & - & 0 & 0 & 0 \\
\hline 2 & - & 0 & 0 & 0 \\
\hline 3 & - & 0 & 0 & 0 \\
\hline 4 & - & 0 & 0 & 0 \\
\hline 5 & - & 0 & 0 & 0 \\
\hline 6 & $3,75(13,75)$ & $0(5,5)$ & $0(0)$ & $0(0)$ \\
\hline 7 & $5(16,25)$ & $0(6,25)$ & $0(0)$ & $0(0)$ \\
\hline 8 & $8(21,25)$ & $0(9)$ & $0(0)$ & $0(0)$ \\
\hline 9 & 0 & 0 & 0 & 0 \\
\hline 10 & 0 & 0 & 0 & 0 \\
\hline 11 & 0 & 0 & 0 & 0 \\
\hline
\end{tabular}

Los valores fuera del paréntesis representan la escala normal de evaluación de toxicidad basada en porciento de tejido necrótico de la caña de azúcar. A su vez, los valores adentro del paréntesis representan la evaluación de toxicidad basada en porciento de blanqueamiento del follaje de la caña de azúcar. Al momento de la primera evaluación de toxicidad de los tratamientos 1 al 5, la caña de azúcar no había emergido, por esta razón no aparecen valores de toxicidad en la columna correspondiente.

Al mezclar el clomazone con la ametrina, se puede incrementar el control de P. niruri y A. spinosus, pero no el de C. rotundus y L. mollis. El control de E. colo$n a$ puede verse reducido, sobre todo cuando se aplican las dosis intermedias y bajas de esta mezcla.

La mezcla de clomazone+ametrina, aplicada en postemergencia a $800+1200 \mathrm{~g} /$ ha tuvo controles de $E$. colona, P. fasciculatum y R. cochinchinensis semejantes o superiores a los obtenidos con diurón+2,4-D $(1600+960 \mathrm{~g} / \mathrm{ha})$ y ametrina+2,4-D (1225 + $650 \mathrm{~g} / \mathrm{ha})$ en postermergencia.

Clomazone+ametrina ocasionó un "blanqueamiento" temporal de las hojas de la caña de azúcar, que a los 15 DDA, ocupaba entre 13,75 y 40\% del área foliar y desapareció completamente entre los 30 y 60 DDA.

\section{LITERATURA CITADA}

CÁMARA, G. M. S.; ARÉVALO, R. A.; ORSI, F.; MAULE, R.F.; PUZZO, R. B. 1995. Eficiência da mistura formulada de clomazone + ametrina no controle das plantas daninhas à cultura da cana-de-açúcar em área de soqueira. p. 436. In: XX Congresso Brasileiro de Plantas Daninhas. Florianópolis, SC. Julho 1995.

CHRISTOFFOLETI, P. J.; CAMPOS, J.A.D.; ORSI,F. 1995. Controle de plantas daninhas em condições de pósemergência inicial na cultura da cana-de-açúcar. p. 202. In: XX Congresso Brasileiro de Plantas Daninhas. Florianópolis, SC. Julho 1995.

DÍAZ, C. J.T.; MASSON, C.G. 1996. Evaluación de flazasulfurón 25 GDA en el control de maleza en el cultivo de caña de azúcar, La Pesca, Ver. México, 1996. p. 69. In: XVII Congreso Nacional de la Ciencia de la Maleza. Ixtapa-Zihuatanejo. Noviembre de 1996

FMC, s/f. Gamit. Boletín Técnico, Caña-de-Açúcar. FMC do Brasil, Indústria e Comercio Ltda. 24 p.

GÓMEZ, K. A.; GÓMEZ, A. A. 1984. Statistical procedures for agricultural research. 2nd. ed. New York, J. Wiley \& Sons. $680 \mathrm{p}$.

MORALES, M. M. D. 1987. Manual de Malezas. México, Instituto para el mejoramiento de la producción de azúcar. $51 \mathrm{p}$.

ORSI, F.; GALLO, P.A. 1995. Avaliação do efeito de doses dos herbicidas clomazone+ametrina e sulfentrazone sobre o crecimento e productividade de soqueira de cana-de-açúcar (Saccharum sp.) em 2 épocas de aplicação. p. 188. In: XX Congresso Brasileiro de Plantas Daninhas. Florianópolis, SC. Julho 1995.

THOMSON, W. T. 1993. Agricultural Chemicals. Book II Herbicides. Revision. Fresno, CA, Thomson Publications. 310 p. 